\title{
Practice effects for visual resolution in the periphery
}

\author{
CHRIS A. JOHNSON and HERSCHEL W. LEIBOWITZ \\ Pennsylvania State University, University Park, Pennsylvania 16802
}

\begin{abstract}
Resolution thresholds at $0^{\circ}$ (fovea), $20^{\circ}, 40^{\circ}$, and $60^{\circ}$ along the horizontal meridian of the temporal visual field revealed a characteristic degradation in visual resolution with increasing stimulus eccentricity. However, substantial individual differences were found, particularly at $40^{\circ}$ and $60^{\circ}$ of eccentricity. Dramatic improvements in peripheral visual resolution occurred over a period of 11 practice sessions, with the time course of practice effects increasing for greater visual field eccentricities. Improvements with practice reduced, but did not eliminate, individual differences. The present visual resolution findings are compared to previous studies of peripheral motion detection and increment thresholds.
\end{abstract}

A number of investigators have examined resolution properties of the peripheral visual field (e.g., Aulhorn \& Harms, 1972; Johnson, Keltner, \& Balestrery, 1978; Kerr, 1971; LeGrand, 1967; Low, 1951; Millodot, Johnson, Lamont, \& Leibowitz, 1975; Weymouth, 1958). Stimulus parameters such as luminance (Kerr, 1971; Low, 1951; Mandelbaum \& Sloan, 1947) and peripheral dioptrics (Green, 1970; Johnson, Leibowitz, Millodot, \& Lamont, 1976; Millodot et al., 1975) have generally been found to have minimal effects on peripheral visual resolution, although Frisen and Glausholm (1975) have reported significant improvements for resolution of interference fringe gratings in the far periphery. In comparison, remarkable improvements in peripheral visual resolution have been reported for various practice and training procedures (Dobrowolski \& Gaine, 1875; Low, 1946, 1951; Saugstad \& Lie, 1964), although there is general disagreement as to the amount and specific characteristics of these practice effects.

The purpose of the present investigation is to extend previous findings by quantitatively evaluating the magnitude and time course of practice effects for visual resolution at various locations in the peripheral visual fields. In addition, it is of interest to compare these results to practice effects for other peripheral visual functions such as motion detection (Johnson \& Leibowitz, 1974), increment thresholds (Aulhorn \& Harms, 1972), and absolute thresholds (Abernethy \& Leibowitz, 1971).

This research was performed in the Department of Psychology at Pennsylvania State University, and was supported by Grant MH08061 from the National Institute of Mental Health. Chris A. Johnson is now at the Department of Ophthalmology, University of California, Davis. Reprint requests should be sent to Chris A. Johnson, Department of Ophthalmology, TB 157, School of Medicine, University of California, Davis, California 95616.

\section{METHOD}

The apparatus has been described in a previous paper (Millodot, et al., 1975). A test stimulus and surround field were presented by means of a two-channel Maxwellian view system (Westheimer, 1966) with an additional fixation target (not in Maxwellian view) provided for peripheral determinations, as shown schematically in Figure 1. The annular surround field (SF) consisted of an opaque ring (inner diameter, $2^{\circ}$; outer diameter, $3^{\circ}$ ) centered within a clear circular portion $\left(10^{\circ}\right)$ of a thin glass sheet, optically positioned at a distance of $1 \mathrm{~m}$ from the subject. Luminance of the clear portions of the surround field was $524 \mathrm{~cd} / \mathrm{m}^{2}$ (retinal illuminance $=2,572 \mathrm{td}$ ). Test stimuli consisted of horizontally oriented sinusoidal gratings (G). limited in size $\left(2^{\circ}\right)$ by a circular field stop (FS) and positioned at an optical distance of $1 \mathrm{~m}$. Space-average luminance of the gratings was $1,118.2 \mathrm{~cd} / \mathrm{m}^{2}$ (retinal illuminance $=5,489 \mathrm{td}$ ), and the exposure duration of the grating $(250 \mathrm{msec})$ was controlled by an electronic shutter (SH). A beam-splitting cube (B) superimposed the test stimulus inside the annulus of the surround field.

Twenty National Bureau of Standards sinusoidal gratings (modulation, .658), successively increasing in spatial frequency by a factor of 1.26 , were used as test stimuli. Refined adjustments in spatial frequency were performed by rotation of the grating about the horizontal axis using a rotary table equipped with a holder. A vernier scale on the table permitted measurements of angular rotation accurate to within $5 \mathrm{~min}$ of arc. Since, at large angular rotations, the spatial distribution of light transmitted by the grating test stimuli departed from a true sinusoidal representation, angular rotations were limited to less than $65^{\circ}$. For the largest stimulus rotation, the dioptric difference between the upper and lower portions of the grating was less than .5 diopter. A brief exposure duration of $250 \mathrm{msec}$ was employed to minimize the possible influence of the field stop (FS) on accommodation during foveal determinations, and to minimize the influence of Troxler's effect for peripheral measurements.

The subject's head was held rigid by a bite-board arrangement which was carefully aligned to the apparatus for each eccentricity examined. A $2.5-\mathrm{mm}$-diam artificial pupil (AP) was located on a thin vertical metal strip which was sufficiently narrow to prevent occluding the fixation stimulus during peripheral determinations. The artificial pupil was employed to control for changes in effective pupil size with eccentric viewing, thereby maintaining a constant retinal illuminance for all determinations. In addition, the artificial pupil served to minimize distortions of the diffraction pattern on the retina produced by the elliptical shape of the natural pupil for large viewing angles (Weale. 1956). 


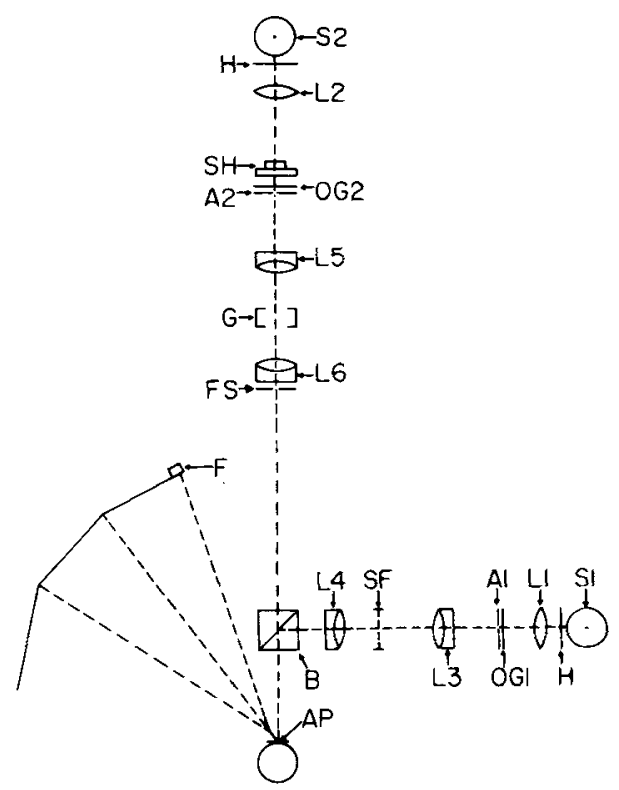

Figure 1. Schemtic representation of the optical system used in this investigation. The symbols refer to the following: S1, S2, tungsten ribbon filament lamps; $H$, heat-absorbing glass filter; A1, A2, apertures which served as secondary light sources; OG1, OG2, opal diffusing glass plates; L1, L2, L3, L4, L5, L6, lenses; SF, surround field, annulus target: B, beamsplitting cube; $G$, sinusoidal grating test stimulus; FS, field stop; $\mathrm{SH}$, electronic shutter; F, fixation stimulus; AP, artificial pupil.

The surround field annulus served to direct fixation for foveal measurements. During peripheral determinations, the fixation stimulus (F) consisted of a dark Landolt ring ( $18 \mathrm{~min}$ of are diameter) centered within a circular background field (41 min of arc diameter) of $15.2 \mathrm{~cd} / \mathrm{m}^{2}$ luminance. This fixation stimulus was moved to eccentricities of $20^{\circ}, 40^{\circ}$, and $60^{\circ}$ for successive determinations, and was located at a distance of $1 \mathrm{~m}$ from the subject.

Visual resolution measurements were performed in four experienced psychophysical observers (20/20 or better acuity, with no apparent ocular abnormalities) at eccentricities of $0^{\circ}$ (fovea), $20^{\circ}$, $40^{\circ}$, and $60^{\circ}$ along the horizontal meridian of the temporal field. Three successive determinations were performed at each eccentricity by means of an interleaved double staircase method (Cornsweet, 1962) with variable step size. Subjects were instructed to report whether the grating lines were present on each trial, and the mean value about which the staircases varied thus defined the $50 \%$ threshold for resolution of the grating. Peripheral refractive errors were not corrected in the present study. A previous paper (Millodot et al., 1975) has reported peripheral refractive error data for each subject, and has shown that corrected and uncorrected peripheral visual acuity values for these subjects are essentially the same.

Eleven test sessions were conducted for each subject, with the duration of each session lasting approximately $1.5 \mathrm{~h}$. The order of presentation of test locations $\left(0^{\circ}\right.$ to $60^{\circ}$, or $60^{\circ}$ to $\left.0^{\circ}\right)$ was alternated for successive test sessions, and the initial presentation order was counterbalanced across subjects. During each session, brief rest periods were given to the subjects following individual threshold determinations. Subjects self-monitored their fixation by means of a blind spot fixation technique (Johnson \& Leibowitz, 1974). Trials during which eye movements occurred were discarded.

\section{RESULTS}

Figure 2 presents resolution threshold values for each subject at $0^{\circ}$ (fovea), $20^{\circ}, 40^{\circ}$, and $60^{\circ}$ of eccen- tricity. The minimum angle of resolution in minutes of arc (defined by the angle subtended by one-half cycle of the sinusoidal grating) is plotted as a function of the number of testing (practice) sessions. An analysis of variance performed on the resolution threshold measurements revealed statistically significant effects for practice sessions $[F(10,352)=29.2$ $\mathrm{p}<.001]$, stimulus eccentricity $[\mathrm{F}(3,352)=1392.6$, $\mathrm{p}<.001]$, subjects $[\mathrm{F}(3,352)=63.2, \mathrm{p}<.001]$, and the interactions of Sessions by Eccentricity $[F(30,352)=6.6, p<.001]$, Eccentricity by Subjects $[\mathrm{F}(9,352)=29.1, \mathrm{p}<.001]$, and Sessions by Eccentricity by Subjects $[F(90,352)=3.5, p<.001]$. The Sessions by Subjects interaction was not statistically significant $[F(30,352)=0.8, p>.10]$. This analysis thus provides statistical support for the interpretations given below.

\section{Eccentricity and Practice}

All subjects displayed an increase in resolution thresholds with increasing stimulus eccentricity, although the magnitude of this effect was reduced with practice. In general, absolute improvements in performance (lower resolution thresholds) were negligible for the fovea and became progressively larger with increasing visual field eccentricity. Relative performance (percent improvement), however, showed a dramatic increase between the fovea and $20^{\circ}$ eccentricity, beyond which little or no changes were evident. Average values of relative improvement for the four subjects were $4.1 \%$ for the fovea, $41.2 \%$ for $20^{\circ}$ $38.3 \%$ for $40^{\circ}$, and $45.7 \%$ for $60^{\circ}$ of eccentricity. In addition, the amount of practice necessary to

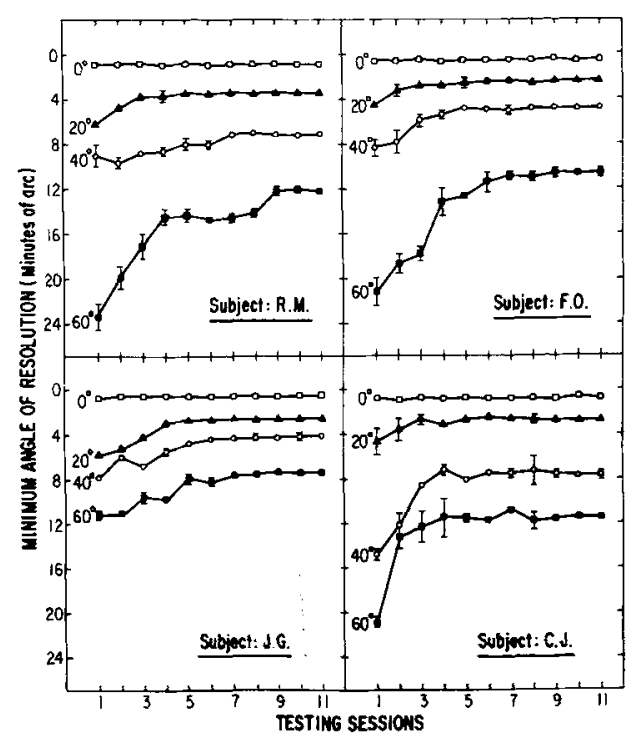

Figure 2. Visual resolution thresholds (minimum angle of resolution in minutes of arc) as a function of the number of testing sessions at $0^{\circ}$ (open squares), $20^{\circ}$ (filled triangles), $40^{\circ}$ (open circles) and $60^{\circ}$ (filled squares) of visual eccentricity. The vertical bars represent \pm 1 standard deviation. Data presented for subjects R.M., J.G., F.O., and C.J. 
achieve optimal performance generally increased for greater stimulus eccentricities (see Figure 2).

\section{Individual Differences}

Resolution thresholds obtained during Session 1 indicate negligible differences among subjects for foveal determinations, but rather large individual differences for measurements at peripheral eccentricities. Practice reduced, but did not eliminate, the substantial individual differences in peripheral visual resolution thresholds. Both the magnitude and time course of practice effects also displayed considerable intersubject variability, particularly at greater stimulus eccentricities (see Figure 2). The remarkable stability in resolution thresholds over the final three testing sessions indicates that individual differences were not due to response inconsistency or withinsubjects variability. No consistent relationships were found for individual differences, peripheral refractive error, and the magnitude and/or time course of practice effects at various eccentricities.

\section{DISCUSSION}

Although significant practice effects for peripheral visual resolution were found in the present study, the improvements are somewhat smaller than those reported by Low $(1946,1951)$. The basis for this difference might be due to the subject populations used in the two studies. Low's subjects were all naive observers with no prior experience in psychophysical testing, whereas the subjects in the present investigation had extensive experience as observers in other psychophysical experiments. Thus, Low's findings may reflect a more generalized psychophysical observer training effect in addition to the specific influence of practice on peripheral visual resolution. Since the results of only the first and last test sessions are reported in previous studies (Low, 1946, 1951; Saugstad \& Lie, 1964), it is difficult to draw comparisons for the time course of practice effects found in the present study. It is implied in prior investigations that approximately 13 (Saugstad \& Lie, 1964) to 25 or 30 (Low, 1946, 1951) sessions are needed to achieve optimal performance. The results of this study suggest that between 4 and 9 test sessions are needed for best performance, depending upon the stimulus eccentricity and the particular observer.

The experimental design employed in this study does not allow direct evaluation of the contributions of "sensitivity" and "criterion" effects to improvements with practice for peripheral visual acuity. However, previous signal detection experiments conducted for peripheral motion thresholds (Johnson, Note 1) indicate that (in experienced psychophysical observers) criterion effects accounted for only a minor portion of the amount of improvement with practice.
We therefore feel that the practice effects reported in the present study primarily reflect changes in sensitivity.

Comparisons of practice effects for peripheral visual resolution with those of other visual functions in the periphery reveal several interesting differences. Absolute thresholds (Abernethy \& Leibowitz, 1971), increment thresholds (Aulhorn \& Harms, 1972), and motion thresholds (Johnson \& Leibowitz, 1974) reach optimal performance levels at all stimulus eccentricities within the first three or four test sessions. Moreover, the time course of practice effects for increment and motion thresholds is relatively constant for all eccentricities beyond $10^{\circ}$. These characteristics are also consistent across subjects. In contrast, peripheral resolution thresholds require a longer period of time to achieve optimum performance levels, exhibit differences in the time course of practice effects across the visual field, and display considerable variation of these characteristics among individuals. Motion and visual resolution thresholds in the periphery also exhibit some similar attributes. Both functions show larger practice effects for the periphery than for the fovea, and the magnitude of improvements with practice are also quite comparable.

From a methodological standpoint, the current findings indicate that unless a stable performance level is reached, practice effects may greatly influence the outcome of experiments involving peripheral vision. On the other hand, these results also show that the visual periphery has a marked potential for improved performance and is highly amenable to training. This suggests that visual tasks demanding considerable input from the periphery may be facilitated through appropriate practice and training procedures.

\section{REFERENCE NOTE}

1. Johnson, C. A. Improvement with practice on peripheral motion discrimination: A look for attentional fluctuations, sensitivity and criterion effects. Paper presented at the 1974 meeting of the Eastern Psychological Association, Philadelphia, Pennsylvania.

\section{REFERENCES}

Abernethy, C. N., \& Leibowitz, H. W. The effect of teedback on luminance thresholds for peripherally presented stimuli. Perception \& Psychophysics, 1971, 10, 172-174.

aulhorn, E., \& Harms, H. Visual perimetry. In L. M. Hurvich \& D. J. Hurvich (Eds.), Handbook of sensory psychology (Vol. VII/4) Visual psychophysics. Berlin: Springer-Verlag. 1972.

Cornsweet. T. N. The staircase method of psychophysics. American Journal of Psychology, 1962. 75. 585-591.

Dobrowolskı, W..\& Gaine, A. Über die Sehschärfe (Formsinn) der Peripherie der Netzhaut. Pflügers Archiv für die gesamte Physiologie, 1875. 12, 411-432.

Frisen. L.. \& Glausholm, A. Optical and neural resolution in peripheral vision. Investigative Ophthalmology, 1975, 14. $52 \times-5,36$. 
GreEn, D. G. Regional variations in the visual acuity for interference fringes on the retina. Journal of Physiology (London). 1970, 207. 351-356.

Johnson, C. A., Keltner, J. L., \& Balestrery, F. G. Effects of target size and eccentricity on visual detection and resolulution. Vision Research, 1978, 18, 1217-1222.

Johnson, C. A., \& Leibowitz, H. W. Practice, refractive error. and feedback as factors influencing peripheral motion thresholds. Perception \& Psychophysics, 1974, 15, 276-280.

Johnson, C. A., Leibowitz, H. W., Millodot, M., \& Lamont, A. Peripheral visual acuity and refractive error: Evidence for "two visual systems"? Perception \& Psychophysics, 1976, 20. $460-462$.

KerR. J. L. Visual resolution in the periphery. Perception \& Psychophysics, 1971, 9, 375-378.

LeGrand, Y. Form and space vision. (M. Millodot \& G. G. Heath, trans.) Bloomington: Indiana University Press, 1967.

Low, F. N. Some characteristics of peripheral visual performance. American Journal of Physiology. 1946, 140, 83-88.
Low, F. N. Peripheral visual acuity. Archives of Ophthalmology, 1951, 45, 577.593.

Mandelbaum, J., \& Sloan, L. L. Peripheral visual acuity. American Journal of Ophthalmology, 1947, 30, 581-588.

Millodot, M.. Johnson, C. A., Lamont, A., \& Leibowitz, H. W. Effect of dioptrics on peripheral visual acuity. Vision Research, 1975, 15, 1357-1362.

SaUgstad, P., \& LIE, I. Training of peripheral visual acuity. Scandinavian Journal of Psychology, 1964, 5, 218-224.

Weale, R. A. Problems of peripheral vision. British Journal of Ophthalmology, 1956, 40,392-415.

Westheimer, G. The Maxwellian view. Vision Research, 1966. 6, 669-682.

WeYMouth, F. W. Visual sensory units and the minimum angle of resolution. American Journal of Ophthalmology, 1958, 46, 102-113.

(Received for publication June 29, 1978; revision accepted February 28, 1979.) 